\title{
State of charge estimation based on adaptive algorithm for Lead-Acid battery
}

\author{
Maamar Souaihia, Bachir Belmadani, Rachid Taleb
}

Electrical Engineering Department, University of Chlef, Algeria

\begin{tabular}{l}
\hline Article Info \\
\hline Article historys: \\
Received Jan 31, 2019 \\
Revised May 1, 2019 \\
Accepted May 21, 2019 \\
\hline
\end{tabular}

\section{Keywords:}

Aadaptive extended Kalman filter

Lead-Acid battery

State of charge

\begin{abstract}
The usage of batteries in recent years has become widespread in many fields e.g. in electric vehicles, energy renewable and stand-alone systems which require a robust approach for estimation of the state of charge (SOC). The SOC represents an important factor to guaranty safe operations. A lot of methods have been used to predict the state of charge. The coulomb counting method is the famous and widely used among them, but have limitation due to its accuracy. Another used approach is the Kalman Filter, which improves the estimation efficiency, to reach a good performance in SOC prediction. The version of adaptive extended Kalman filter (AEKF) technique is applied in this paper. This paper presents an experimental performance of technique of Kalman filter, for solving the problem of accurate SOC. The method is used to compute the terminal voltage in such a way to estimate the SOC. The proposed algorithm is based on preselected Thevenin model after the identification of its parameters. It has been used to predict the SOC based on nonlinear equations, and evaluation of the approach is verified with the experimental results. The final results signify that the estimation matched with the proposed model and the algorithm is performed optimally, thus the maximum soc estimation error is the finest.
\end{abstract}

Copyright (C) 2019 Institute of Advanced Engineering and Science. All rights reserved.

\section{Corresponding Author:}

Maamar Souaihia,

Electrical Engineering Department,

Hassiba Benbouali University of Chlef, LGEER Laboratory

BP. 78C, Ouled Farès 02180, Chlef, Algeria.

Email: maa.souaihia@gmail.com

\section{INTRODUCTION}

In recent years, applications of the battery become widespread because it is friendly to the environment. Many advantages are available in energy transforming from battery, which provides benefits in stakeholders and integration of renewable sources including solar and wind. The main objective of renewable sources is to take advantages of a question of reliability of the systems supply, bring vitality to the demand response of electric storage, and also addressing the issues of eco-friendly and economic $[1,2]$.

Due to the modern technologies used in making the batteries and the interest in energy storage techniques and trying to lower the price of the solar panels, the reliability of the electronic energy can be improved by the usage of the energy of the battery. In addition, the battery storage devices response in a short period of time. Hence, they can be distributed in various sizes and also can be evaluated by fast control systems [3].

In batteries, the electrical energy come from generating a direct current through chemical reactions. The cell acts as a storage device since it is influenced by the internal and external factors such as temperature and the electronic load. A mathematical model of the battery is necessary to match the chemical reaction into electrical behaviour which makes the battery simple to use in embedded systems, thereby for more robust in estimation [4]. 
In the literature, various methods are proposed for SOC estimation. Each technique is different in accuracy than others but, the most are complicated or expensive. The main goal of this paper is to design a system capable to estimate the SOC of a battery, simple and costless. SOC estimation method using spectroscopy of the impedance [5] is a very expensive approach, because it needs impedance meter, a discharge test under controlled conditions. The open circuit voltage (OCV) [6-12] or the internal resistance [13] method require a relaxation period of time to reach the equilibrium voltage after disconnecting from the system in each step, and the estimation due according to the relationship between the OCV and the SOC. The coulomb counting [14-16] is the most popular method. It uses the current integral over time, is simple and needs fewer complex computations. However, this method has the disadvantage of cumulating the errors due to uncertain disturbances. In literature exist a lot of algorithms of intelligence, such as artificial neural networks (ANN) and fuzzy logic [17-19] which have been also employed to estimate the SOC. These methods do not require an initial knowledge about the battery; thus, they support all types of batteries. They have been applied and gave excellent estimation. However, it is time consuming and impossible the training data cover all the possible loading conditions.

Numerous efforts have been concentrated on model-based for estimation, including The Kalman filter (KF) [20-23] and sliding mode observer [24, 25]. These methods are self-correcting and require a high computation. They are more popular, suitable for tracking, and applicable for online estimation. There are a lot of versions of the Kalman filter, to expand for nonlinear systems the extended KF (EKF) [26-28], unscented KF (UKF) [29-32] or the adaptive extended KF (AEKF) [33, 34] have been developed. The AEKF algorithm is an algorithm strongly depends on the predetermined variables of the model battery, such as system noise, covariance matrix. It is updating iteratively the process and measurement noise covariance.

The present paper is based on the first order equivalent circuit model of an AEKF algorithm to predict the SOC. A Lead Acid battery is carried out for the experimental data. To evaluate the performance of the estimation, the battery tested with various C-rate pulse discharges and a simple discharge that result in estimation and model based stochastic observer to enhance the estimated SOC against the errors and challenge of reduce the error covariance. The obtained results prove that tracking accuracyof AEKF-based model for the terminal voltage and the estimation of the SOC are excellent.

In this paper, an adaptive algorithm (AEKF) based on combined battery equivalent circuit has been proposed for SOC estimation. The main advantage of the AEKF is that the accuracy of estimation is ensured in the presence of modeling errors and parameters uncertainties. This is achieved by dynamically adjusting the covariance of measurement and error while guaranteeing the prediction of states, thereby enhancing the SOC estimation robustness.

The rest of this paper is outlined as follows: In the coming section, an overview of the battery model selected, and then we explain the parameterization of the proposed model. In section three, the description of the SOC estimation based on AEKF where the algorithm clarified. A discussion of the experimental results is then illustrated in section four. Finally, the conclusions of the paper are given in the last section.

\section{BATTERY MODEL}

There are several factors that affect the operation of a battery, the main aim of modelling the cell is to predict the behaviour of it by setting that factors in consideration. There are a wide of variety of models with differences degree of complexity, the simplest models are the mathematical models based on simple relations.

To estimate the SOC, the battery is considered as a nonlinear system which has its own dynamics, can be represented by a state space form, their parameters can be calculated as follows:

$$
\begin{aligned}
& \dot{x}=f(x, u)+w \\
& y=g(x, u)+v
\end{aligned}
$$

where $x$ represents the state vector such a SOC, it affected by the system input $u$, which significant the current operation of the system. And $y$ is denoting the measurable output of the system. Also, the $w$ is representing the noise which affects on the system state, in opposition, the $v$ does not affect on the system but it reflects in the output measurements. In different way, $w$ and $v$ are the white Gaussian noise with zero mean and covariance $Q$ and $R$ respectively,$f(x, u)$ and $g(x, u)$ are two functions links the model to the cell specification, moreover the dynamics of the system and the static effects illustrated in (1).

\subsection{Soc definition}

State of charge is a percentage expressing the charge stored in the battery, as a gauge changes in the extent of $0<\mathrm{SOC}<1$. It is calculated by the relativity of instance capacity and the sum of upcoming and incoming Amperes, the ratio of available capacity is given by:

Indo. J. Elec. Eng. \& Inf, Vol.7, No. 2, June 2019: 229- 240 


$$
S O C_{t}=S O C_{0}-\frac{1}{C_{n}} \int I_{L} d \tau
$$

where $S O C_{t}$ and $S O C_{0}$ are the present SOC and the initial value of SOC respectively, $I_{L}$ is the load current (considered positive for discharge and negative for charge), $C_{n}$ is the nominal capacity depending on the ageing of the battery and which may differ from the rate of discharge. The (2) also can transform into discrete time as follow:

$$
S O C_{K}=S O C_{K-1}-\frac{I_{L, K} \Delta t}{C_{n}}
$$

where, $\Delta \mathrm{t}$ is the sampling time in predefined period, the (3) gives a basis to calculate the SOC with known inputs and outputs at sampling time Kth in state equation format, which is an additional component in the state vector used to build the model of the battery.

\subsection{State space model}

Physical explanation can be expressed by electrical circuit components and include also the dynamics effects of the battery, various models used to fit the real experiment results, due to the chemical reaction and the effects of relaxation, all models required precision and suitable behaviour. To build an accurate model which fit all the data of experiment and the several changes, the first order equivalent circuit model (Thevenin Model) is illustrated in Figure 1.

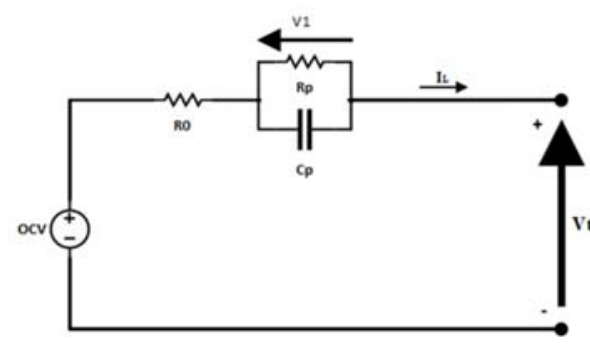

Figure 1. Schematic of the first order model

The battery model selected is formed by a branch of parallel resistor and capacitor combination $R_{p}$, $C_{p}$ respectively simulate the polarization and the transient dynamics, an OCV is the battery open circuit voltage, and a resistor $R_{0}$ represent the ohmic resistance of the electrolyte and electrode, finally $V_{t}$ is the battery terminal voltage. The schematic equivalent circuit can be expressed be equations as followsb (4) and (5):

$$
\begin{aligned}
& V_{t}=O C V-R_{0} I_{L}-V_{p} \\
& \dot{V}_{1}=\frac{I_{L}}{C_{p}}-\frac{V_{1}}{C_{p} R_{p}}
\end{aligned}
$$

The model can suit a good quality if the parameters are calculated accurately from the test data. To identify the parameters, a specific test conducted.

In order to apply the battery model selected in AEKF algorithm, the battery equations converted as state-space equations, in this transformation, the SOC and the voltage across the branch $R_{p} C_{p}$ are chosen as the state variable. SOC is expressed as (2). The final state equation for first order equivalent circuit model can be formulated as represented in (6) and (7).

$$
\begin{aligned}
& x=\left[\begin{array}{ll}
\operatorname{SOC} V_{1}
\end{array}\right]^{T} \\
& \dot{x}=\left[\begin{array}{cc}
0 & 0 \\
0 & \frac{-1}{C_{p} R_{p}}
\end{array}\right] x+\left[\begin{array}{c}
\frac{-1}{C_{n}} \\
\frac{1}{C_{p}}
\end{array}\right] I_{L}
\end{aligned}
$$

After the transformation to the state-space equation at time step $k$, the governing equations of the whole system can be denoted as (8) and (9) by involving the interval of time $\Delta \mathrm{t}$ : 


$$
\begin{aligned}
& x_{k+1}=\left[\begin{array}{cc}
1 & 0 \\
0 & 1-\frac{\Delta t}{C_{p} R_{p}}
\end{array}\right] x_{k}+\left[\begin{array}{c}
\frac{-\Delta t}{C_{n}} \\
\frac{\Delta t}{C_{p}}
\end{array}\right] I_{L, k} \\
& V_{t, k}=\left[\begin{array}{ll}
\frac{\partial O C V}{\partial s o c} & -1
\end{array}\right] x_{k}+\left[-R_{0}\right] I_{L, k}
\end{aligned}
$$

\subsection{Parameters extraction}

For the first order equivalent circuit model indicated in Figure 1, the value of parameters $\left\{R_{0}, R_{p}, C_{p}\right\}$ and the relation between OCV and SOC must be determined first. In order to identify these parameters, some experience carried out in a lead-acid battery. In this paper, 12V, 22 Ah lead acid battery is used. An electronic load with specific amperage and power is used to discharge the battery. An Arduino mega 2560 was used as a data acquisition card to collect and store the measurement data to the computer. Matlab/Simulink has utilized the battery data obtained from Arduino (Figure 2). The sampling rate is set to 1 second; a higher sampling rate is not preferable because of the memory space.

In this paper, the battery tested is conducted for the modeling purpose firstly, thereby the first test is the pulse discharge experiment. The test is settled in order to extract the transient response and the behavior of the battery. The pulse discharge test involves sequences of discharging current and rest period of time as presented in Figure 3.

A battery testing bench is constructed to settle the experiment, and it consists of a power supply, electronic loads and switches for safety. The testing platform is illustrated in Figure 2. The testing prototype can control charging / discharging battery, the data sampling stored into PC host via interface program designed by using the MATLAB/Simulink software.

At the beginning of the experiment, the battery is fully charged, and then the battery is discharged with a specific constant current $1 \mathrm{C}$ to reduce $10 \%$ of the state of charge. After that, the battery is taken to rest for a period of time to reach its equilibrium voltage before the next discharge. The cycle of discharge-rest repeated until the battery voltage drop to $10.5 \mathrm{~V}$. Various currents of C-rate are used to test the dynamic behaviour of the battery.

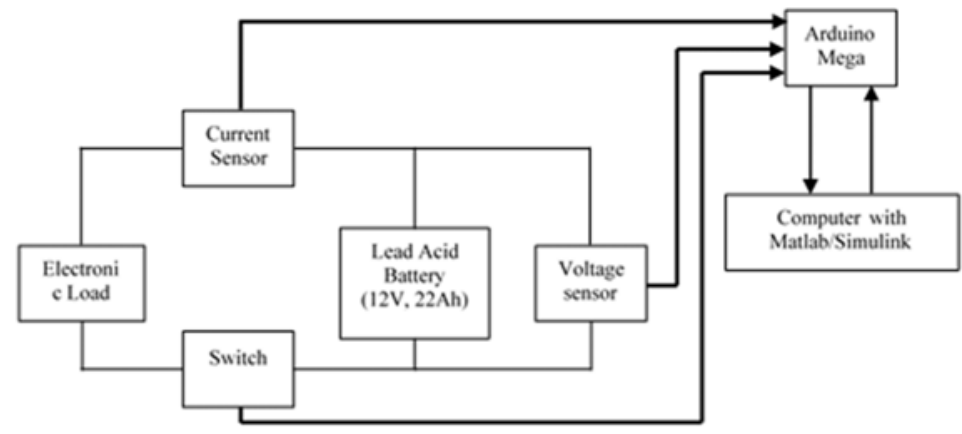

Figure 2. Schematic structure of the experiment
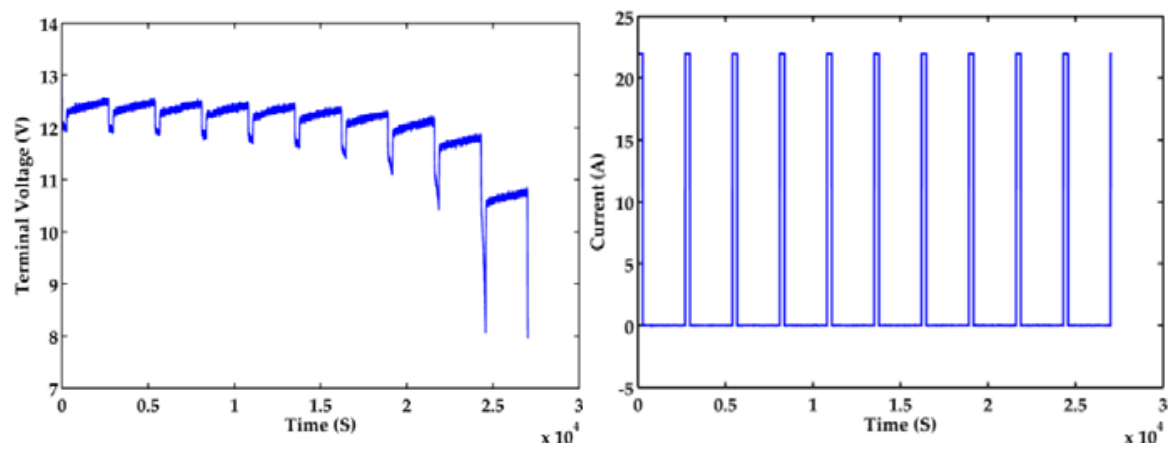

Figure 3. Voltage and current profiles for the pulse discharge test 


\subsection{OCV-SOC relationship}

At the equilibrium state, the terminal voltage defined as the open circuit voltage of the battery. The value of SOC is related to the value of OCV. In this work, the OCV is defined from the test pulse discharge when the battery has a relaxation time. The relationship between OCV and SOC is presented in Figure 4. As shown in the illustration has a curve within the SOC range.

By using the curve fitting toolbox in Matlab, a polynomial of the sixth-order equation can be expressed that relationship as denoted in (10) and its parameters are listed in Table 1.

$O C V=p_{1} S O C^{5}+p_{2} S O C^{4}+p_{3} S O C^{3}+p_{4} S O C^{2}+p_{5} S O C+p_{6}$

Table 1: Parameters of (10)

\begin{tabular}{cr}
\hline Coefficients & \multicolumn{1}{c}{ Value } \\
\hline $\mathrm{p}_{1}$ & 10.897 \\
$\mathrm{p}_{2}$ & -18.852 \\
$\mathrm{p}_{3}$ & 13.692 \\
$\mathrm{p}_{4}$ & -10.089 \\
$\mathrm{p}_{5}$ & 6.9317 \\
$\mathrm{p}_{6}$ & 9.9758 \\
\hline
\end{tabular}

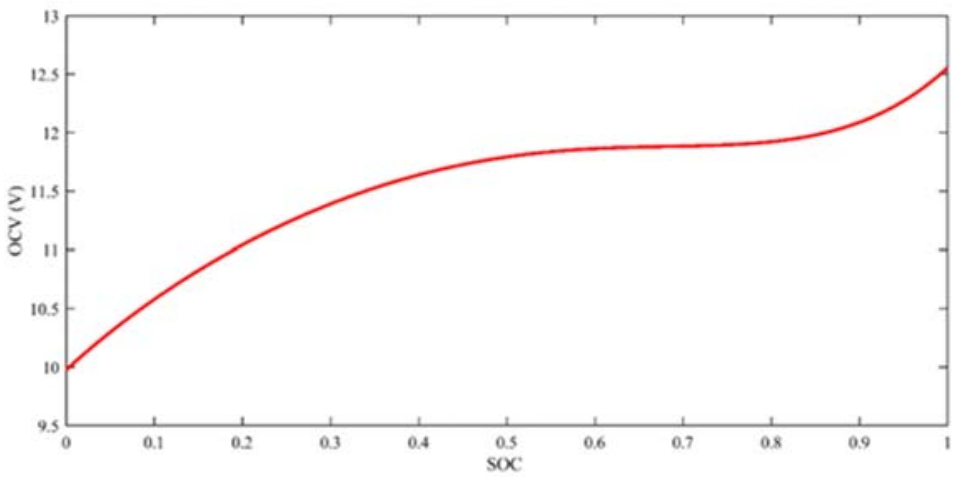

Figure 4. The relationship between open circuit voltage and state of charge

\subsection{Series resistance and $\mathrm{RC}$ branch}

The transient voltage response for pulse discharge and rest are presented in Figure 5. The series resistance can be calculated in the pulse discharge, when the current flow into the battery. And there are a shut down on the potential from the open circuit voltage as shown in Figure 5(a). The equation can be expressed as (11) [24]:

$$
R_{0}=\operatorname{moy}\left(\left|\frac{V_{1}-V_{2}}{I_{L}}\right|+\left|\frac{V_{3}-V_{4}}{I_{L}}\right|\right.
$$

when we compare the obtained value of $R_{0}$ with the value given by the manufacturer, we observe that the value is changed and there is a difference than the fresh battery. The comparison between them helps us to know the state of health of the cell.

To obtain the more accurate model, the parameters must all predefined offline. The identification of the branch parallel RC can be improved by metaheuristic algorithms or by genetic algorithm. In this paper, a Firefly algorithm [35] is used to find the optimal $\tau$ with the objective function:

$$
\left\{\begin{array}{c}
\min \left\{f\left(\hat{x}_{j}^{g}\right)\right\} \\
f(\hat{x})=\frac{1}{N} \sum_{i=1}^{N}\left(V_{t, j}-\hat{V}_{t, k}\left(\hat{x}_{j}^{g}\right)\right)
\end{array}\right.
$$

where $\hat{x}_{j}^{g}$ is the predicted value of the current population at $x_{j}$ generation at $g$ and $j$ is the current position of the individual. Moreover, Nis the estimation length. $\widehat{\mathrm{V}}_{\mathrm{t}, \mathrm{k}}$ represent the estimated value of the terminal voltage. Where, $=\tau$. Or we can choose to estimate the capacitance as: $=\tau / R_{p}$. As illustrated in Figure $5(b)$. 


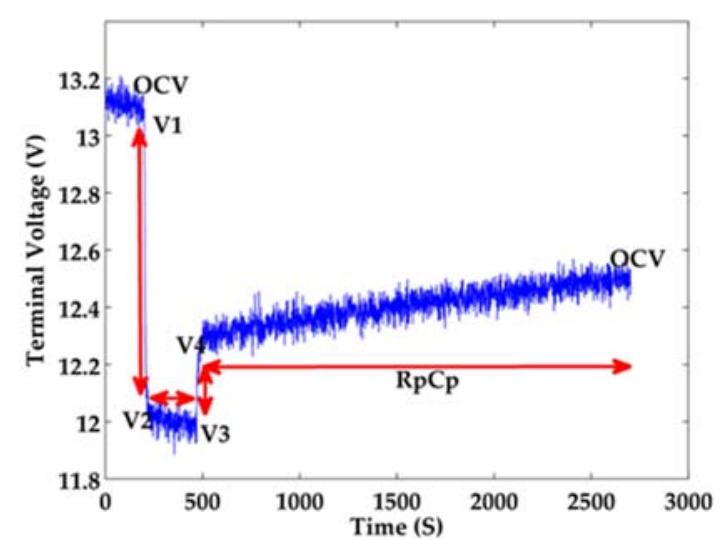

(a)

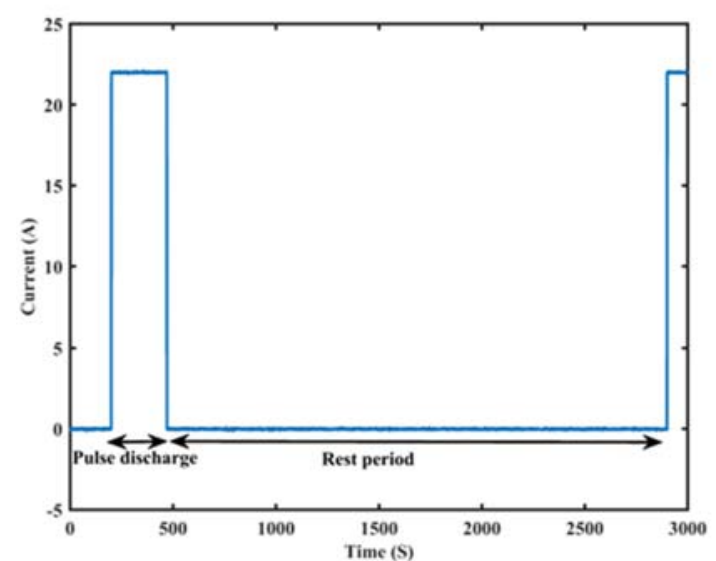

(b)

Figure 5. Transient voltage response; (a) for pulse discharge, (b) pulse of discharge Current.

\section{AEKF ESTIMATION APPROACH}

The Kalman filter is a mathematical function provides estimating states with iterative steps, in a way to minimize the mean squared error. This technique has been providing performance efficiency in the field of estimation parameters and the state transition. It has been used in multiple tracking domains.

The state space model of the battery noted in (10) and (11) are used to predict the SOC. Since the EKF has been a designed for nonlinear system as expressed in (14), where the $\mathrm{k}$ is the time sampling, $\mathrm{x}_{\mathrm{k}}$ is the nonlinear state, $\mathrm{u}_{\mathrm{k}}$ and $\mathrm{y}_{\mathrm{k}}$ are the input and the output respectively.

$$
\left\{\begin{array}{c}
x_{k+1}=f\left(x_{k}, u_{k}\right)+w_{k} \\
y_{k}=g\left(x_{k}, u_{k}\right)+v_{k}
\end{array}\right.
$$

where, the $w_{k}$ and $v_{k}$ are discrete processnoise with covariance matrices $\mathrm{Q}$ and $\mathrm{R}$ respectively.

In this paper, the nonlinear state is denoted as (8), the battery current has been defined as the input, and the terminal voltage has been defined as the output. For nonlinear system the Jacobean matrix of partial derivatives is applied on function $f$ and $g$, state space equations are transformed as (16).

$$
\left\{\begin{array}{c}
\boldsymbol{x}_{\boldsymbol{k}+\mathbf{1}}=A_{k} \boldsymbol{x}_{\boldsymbol{k}}+B_{k} u_{k}+w_{k} \\
\boldsymbol{y}_{k}=C_{k} \boldsymbol{x}_{k}+D_{k} u_{k}+v_{k}
\end{array}\right.
$$

And the matrices $A_{k}, B_{k}, C_{k}$ and $D_{k}$ are:

$$
\left\{\begin{array}{l}
A_{k}=\frac{\partial\left(x_{k}, u_{k}\right)}{\partial \mathrm{x}_{k}} \\
B_{k}=\frac{\partial\left(x_{k}, u_{k}\right)}{\partial \mathrm{x}_{k}} \\
C_{k}=\frac{\partial\left(x_{k}, u_{k}\right)}{\partial \mathrm{u}_{k}} \\
D_{k}=\frac{\partial\left(x_{k}, u_{k}\right)}{\partial \mathrm{u}_{k}}
\end{array}\right.
$$

As expressed in (10) and (11), the matrices can be rewritten as represented in (16) - (19) respectively.

$$
\begin{aligned}
& A_{k}=\left[\begin{array}{cc}
1 & 0 \\
0 & 1-\frac{\Delta t}{C_{p} R_{p}}
\end{array}\right] \\
& B_{k}=\left[\begin{array}{l}
\frac{-\Delta t}{C_{n}} \\
\frac{\Delta t}{C_{p}}
\end{array}\right]
\end{aligned}
$$




$$
\begin{aligned}
& C_{k}\left[\begin{array}{ll}
\frac{\partial O C V}{\partial S O C} & -1
\end{array}\right] \\
& D_{k}=\left[-R_{0}\right]
\end{aligned}
$$

$A_{k}$ and $C_{k}$ are the derivative matrices of the function $f(x, u)$ and $g(x, u)$ respectively to the state vector. $B_{k}$ and $D_{k}$ are the derivative matrices of the system input of the two function respectively. a good fitting of the Kalman filter is depending on the $Q_{k}$ and $R_{k}$ matrices covarianceof processand measurements noises. So, bad initial noise information will cause a fail performance and badestimation.

The initialization of AEKF algorithm is expressed in follow:

$$
\begin{aligned}
& \mathrm{K}=0 ; \\
& x_{0}^{+}=E\left[x_{0}\right] \\
& P_{0}^{+}=E\left[\left(x_{0}-\hat{x}_{0}^{+}\right) \cdot\left(x_{0}-\hat{x}_{0}^{+}\right)\right] \\
& x_{k}=x_{k-1}+\dot{x}_{k} \Delta t
\end{aligned}
$$

the computation of AEKF consists of six steps.

(i) Innovation:

$$
e_{k}=y_{k}-g\left(\hat{x}_{k}^{-}, u_{k}\right)
$$

(ii) Adaptivelaw:

The covariance has been iteratively updating. $Q_{k}$ and $R_{k}$ have been estimated respectively.

$$
\begin{aligned}
& H_{k}=\frac{1}{M} \sum_{k-M+1}^{k} e_{i} e_{i}^{T} \\
& R_{k}=H_{k}-C_{k} P_{k}^{-} C_{k}^{T}
\end{aligned}
$$

(iii) State estimation covariance

$$
P_{K}^{-}=\left(I+A_{k} \Delta t\right) P_{k-1}\left(I+A_{k} \Delta t\right)^{T}+Q_{k}
$$

(iv) Kalman gain

$$
K_{k}=P_{k}^{-} C_{k}^{T} *\left(C_{k} P_{k}^{-} C_{k}^{T}+R_{k}\right)^{T}
$$

(vi) State estimate update

$$
\hat{x}_{k}^{+}=\hat{x}_{k}^{-}+K_{k} e_{k}
$$

(vii) Update state covariance

$$
\begin{aligned}
& Q_{k}=K_{k} H_{k} K_{k}^{T} \\
& P_{k}^{+}=\left(I-K_{k} C_{k}\right) P_{k}^{-}\left(I-K_{k} C_{k}\right)^{T}+K_{k} R_{k} K_{k}^{T}
\end{aligned}
$$

where $I$ is identity matrix. In this step, error covariance is estimated and updated. The computing is then repeating again from (i) to (vii) until reach all the collected data.

\section{RESULTS AND VALIDATION}

In this section, the sate space equations for the first order equivalent circuit model are applied for the prediction of SOC. The validation of AEKF state of charge estimation is checked by the comparison of the experimental SOC and the estimated SOC. In this part, the experiment SOC is measured by counting coulomb method as denotedin (3). The method is tested with predefined value of SOC.

\subsection{Initial condition and noise covariance}

The initial value of the state $\left(x_{0}\right)$ has been shown randomly, the error covariance $\left(P_{0}\right)$, and process noise covariance $(\mathrm{Q})$. Also, the sensor error covariance $(\mathrm{R})$, all are chosen as cited in follow: 


$$
\left\{\begin{array}{c}
x_{0}=[600]^{T} \\
P_{0}=\left[\begin{array}{cc}
100 & 0 \\
0 & 0.01
\end{array}\right] \\
Q=\left[\begin{array}{cc}
0.001 & 0 \\
0 & 0.001
\end{array}\right] \\
R=[0.02]
\end{array}\right.
$$

\subsection{Validation of AEKF state of charge estimation}

A discharge test has been carried out to evaluate the performance of the designed AEKF SOC estimation. The experiment values of the SOC are checked to the estimated illustrated in Figure 6 (a). Moreover, the initial SOC for the algorithm has deflected to the real SOC. The algorithm is already able to estimate the accurate value of SOC in a short time. In addition to, the model output from the AEKF prediction is also excellent in tracking and suit the measurement value of the battery terminal voltage as presented in Figure 8(a).

The AEKF technique furthermore validates the pulse discharge tests. The comparative analysis shows the good tracking and matching between experiment and simulated results. The results of simulation and experiment for the pulse discharge test are shown in Figure 6(b), Figure 6(c), Figure 6(d) respectively to the rate of discharge of $0.4 \mathrm{C}, 0.5 \mathrm{C}$ and $1 \mathrm{C}$.

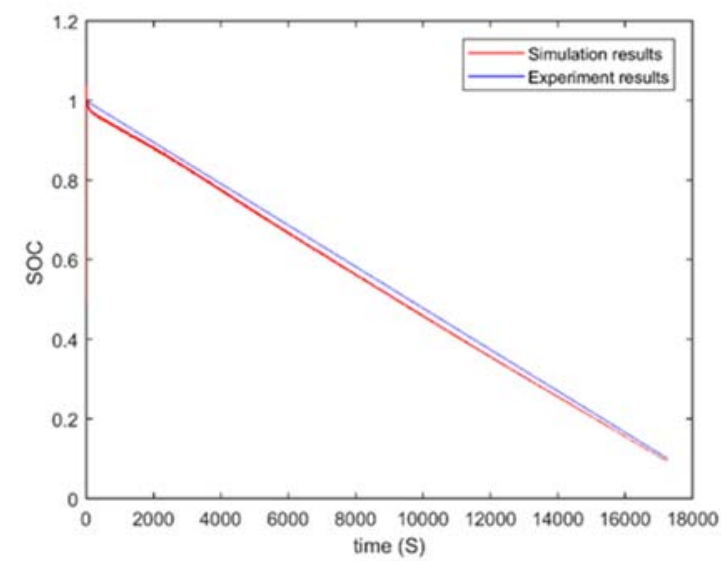

(a)

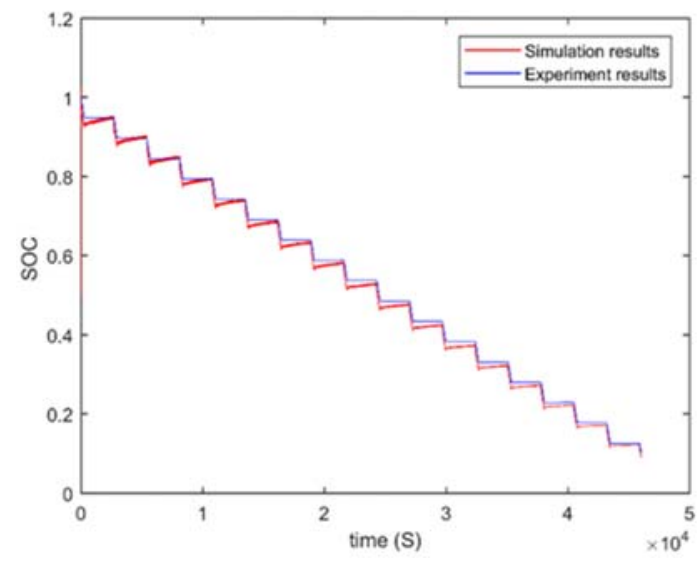

(c)

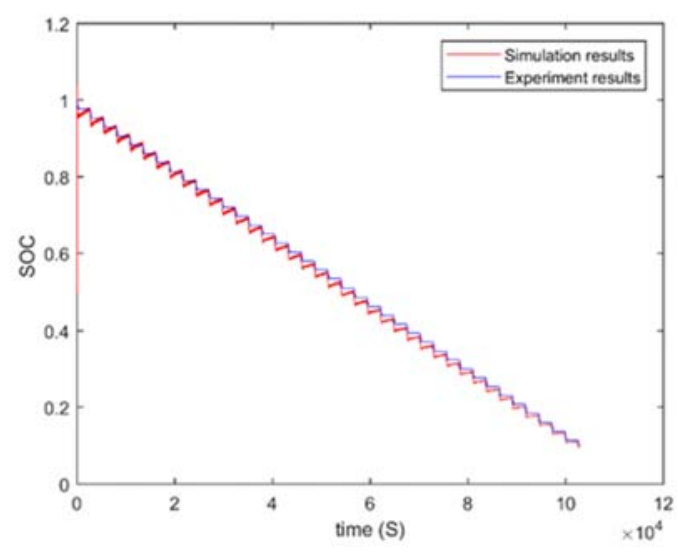

(b)

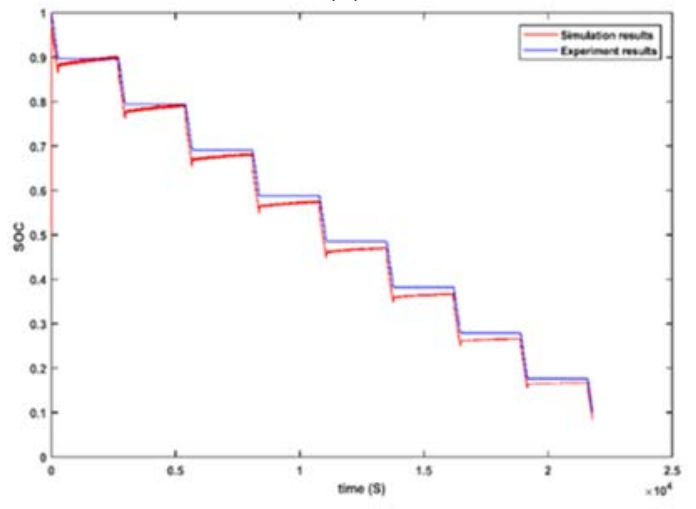

(d)

Figure 6. Comparison between experimental SOC and AEKF estimated SOC; (a) discharge test, (b) 0.4C pulse discharge test,(d) $0.5 \mathrm{C}$ pulse discharge test, and (d) $1 \mathrm{C}$ pulse discharge test.

The results we obtained with AEKF SOC estimation very excellent and appears clearly that the estimation close to the real measurement of the SOC. The algorithm gives a better performance, and the results are very interesting as illustrated in Figure 7, the error is less than $2 \%$. Moreover, it is very accurate towards the measurements noise and poor initialisation of the SOC.

Indo. J. Elec. Eng. \& Inf, Vol.7, No. 2, June 2019: 229- 240 


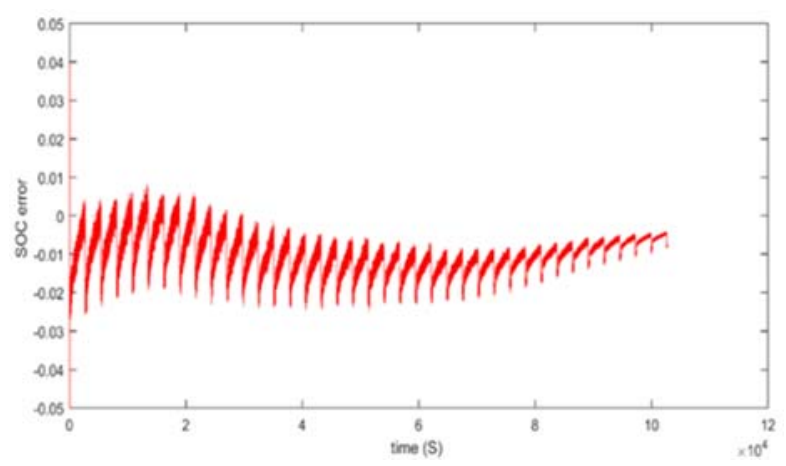

Figure 7. The error of estimation of the SOC for a pulse discharge of $0.5 \mathrm{C}$

By comparing these two results of tracking the output of experiment and the algorithm, we find that the AEKF can reach almost the same asthe experiment and are very close to each other. As presented in Figure 8(b), Figure 8(c) and Figure 8(d). By comparing these two results of tracking the output of experiment and the algorithm, we find that the AEKF can reach almost the same asthe experiment and are very close to each other. As presented in Figure 8(b), Figure 8(c) and Figure 8(d). By comparing these two results of tracking the output of experiment and the algorithm, we find that the AEKF can reach almost the same asthe experiment and are very close to each other. As presented in Figure 8(b), Figure 8(c) and Figure 8(d).

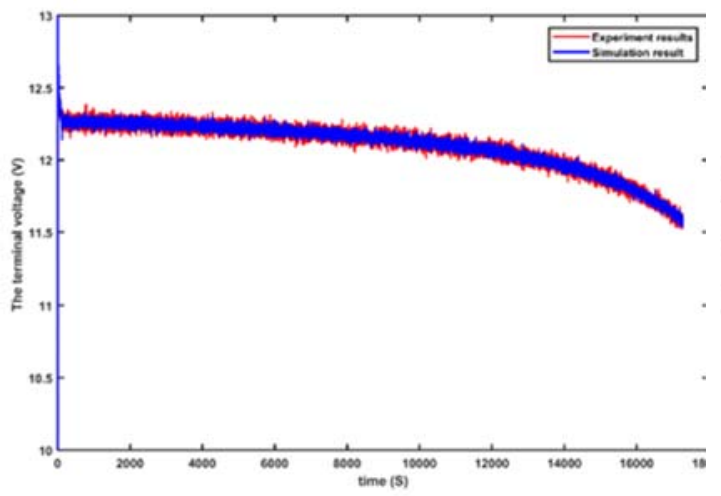

(a)

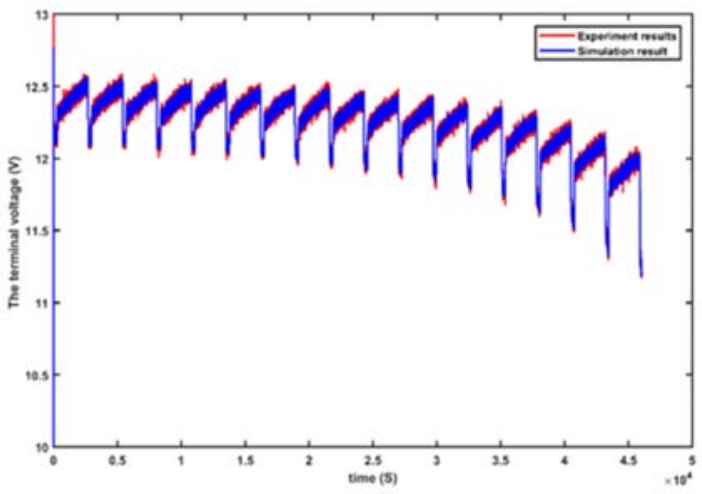

(c)

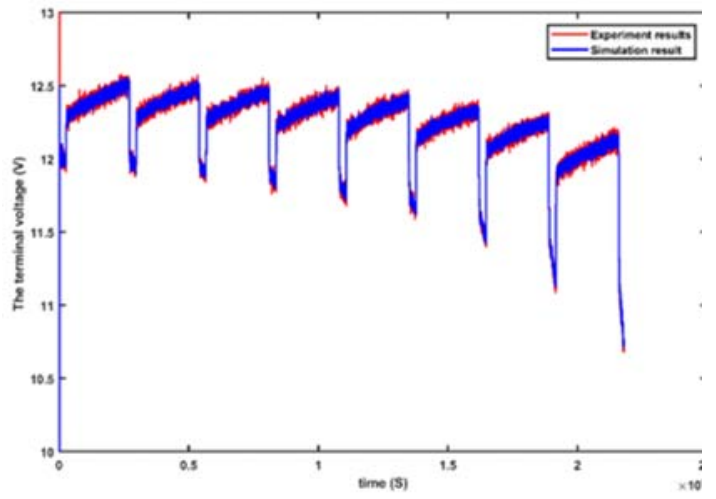

(b)

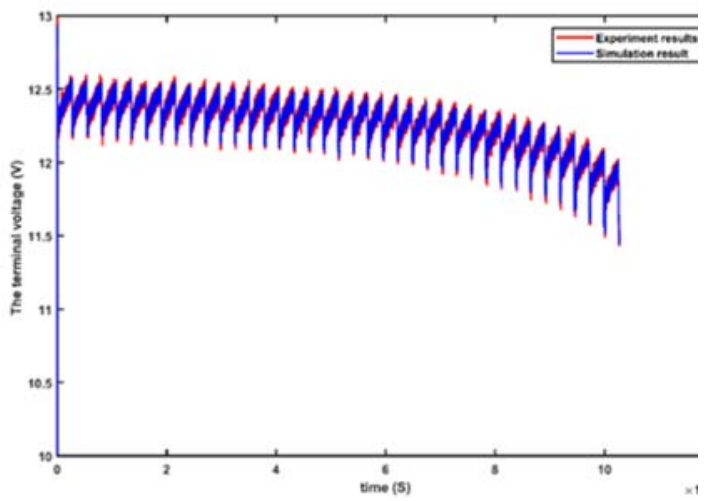

(d)

Figure 8. Comparison between experimental and simulated terminal voltage; (a) discharge test, (b) $0.4 \mathrm{C}$ pulse discharge test, (d) $0.5 \mathrm{C}$ pulse discharge test, and (d) $1 \mathrm{C}$ pulse discharge test. 
The importance of this model that is evident under dynamic condition. And the battery operates always under a dynamic condition of current or temperature. In fact, the adoption of this algorithm proposed with Thevenin model allows us to predict the terminal voltage with better accuracy, actually the error of estimation about 4\%, as presented in Figure 9.

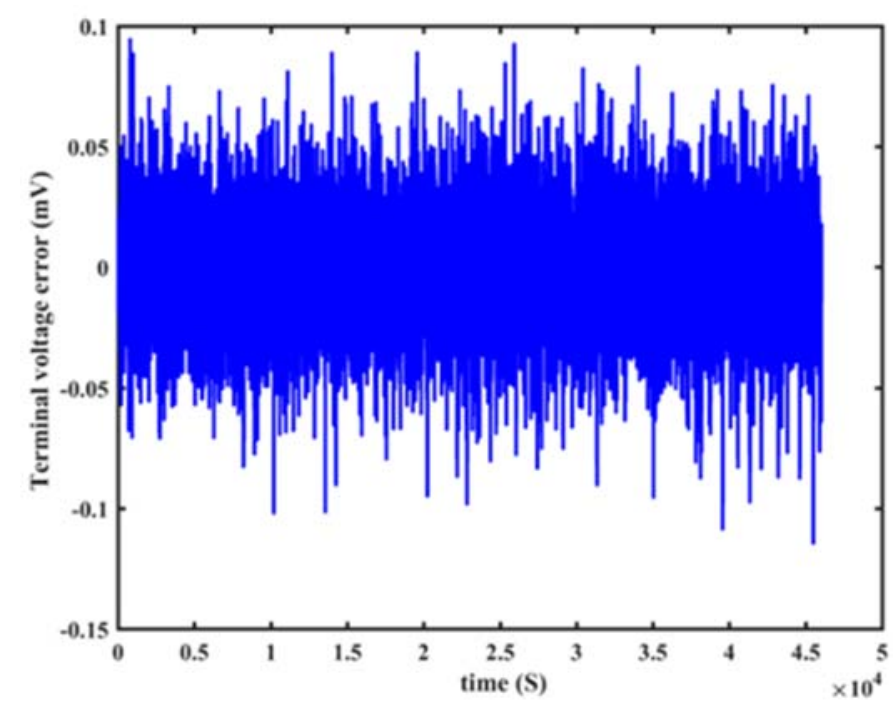

Figure 9. Error of estimation of the terminal voltage for pulse discharge $0.5 \mathrm{C}$ current

\section{CONCLUSION}

This paper presents a brief performance of the AEKF algorithm for solving the optimal estimation of the state of charge problem for battery tracking terminal voltage with the objective of improving the output voltage prediction. The technique of estimation and the extraction of the model parameters operates with nonlinear equations in the way to use the first order equivalent circuit model.

In order to obtain a robust model for control. From many techniques cited in literature, the proposed technique is simple and could be applied to predict and update the parameters of the model. Thus, the possibility to get a strong model fit the experimental data, deal with dynamic conditions, costless and easy to implement.

The algorithm has been tested and performed, showing good suitable results. The simulation results approaching to the experimental data which validate the method. Moreover, the simulation of SOC error always less than $2 \%$ which demonstrate that the purposed method has been used for the estimation well robust and accurate, in addition, adopted as self-correction capability to initial errors.

\section{REFERENCES}

[1] Y. Li, P. Chattopadhyay, A. Ray, and C. D. Rahn, "Identification of the battery state-of-health parameter from inputoutput pairs of time series data," Journal of Power Sources, vol. 285, pp. 235-246, 2015.

[2] A. Mezouari, R. Elgouri, M. Igouzal, M. Alareqi, K. Mateur, H. Dahou, and L. Hlou, "A New Photovoltaic Energy Sharing System between Homes in Standalone Areas," International Journal of Electrical and Computer Engineering (IJECE), vol. 8, p. 4855, 2018.

[3] K. S. M. L. Rimon, S.I. Sulaiman, AM Omar, "System protection for lithium-ion batteries management system: A review," Indonesian Journal of Electrical Engineering and Computer Science (IJEECS), vol. 13, 2019.

[4] A. Zainuri, U. Wibawa, M. Rusli, R. N. Hasanah, and R. A. Harahap, "VRLA battery state of health estimation based on charging time," TELKOMNIKA (Telecommunication, Computing, Electronics and Control), vol. 17, pp. 1577-1583, 2019.

[5] M. Galeotti, C. Giammanco, L. Cinà, S. Cordiner, and A. Di Carlo, "Synthetic methods for the evaluation of the State of Health $(\mathrm{SOH})$ of nickel-metal hydride $(\mathrm{NiMH})$ batteries," Energy conversion and management, vol. 92, pp. 1-9, 2015.

[6] T. Zhao, J. Jiang, C. Zhang, K. Bai, and N. Li, "Robust online state of charge estimation of lithium-ion battery pack based on error sensitivity analysis," Mathematical Problems in Engineering, vol. 2015, 2015.

[7] K.-S. Ng, C.-S. Moo, Y.-P. Chen, and Y.-C. Hsieh, "State-of-charge estimation for lead-acid batteries based on dynamic open-circuit voltage," in 2008 IEEE 2nd International Power and Energy Conference, pp. 972-976, 2008.

Indo. J. Elec. Eng. \& Inf, Vol.7, No. 2, June 2019: 229- 240 
[8] C. Zhang, J. Jiang, L. Zhang, S. Liu, L. Wang, and P. Loh, "A generalized SOC-OCV model for lithium-ion batteries and the SOC estimation for LNMCO battery," Energies, vol. 9, p. 900, 2016.

[9] S. Jiang, "A parameter identification method for a battery equivalent circuit model," SAE Technical Paper, pp. 0148-7191, 2011.

[10] T. Ting, K. L. Man, C.-U. Lei, and C. Lu, "State-of-Charge for Battery Management System via Kalman Filter," Engineering Letters, vol. 22, 2014.

[11] R. Jackey, M. Saginaw, P. Sanghvi, J. Gazzarri, T. Huria, and M. Ceraolo, "Battery model parameter estimation using a layered technique: an example using a lithium iron phosphate cell," SAE Technical Paper, pp. 0148-7191, 2013.

[12] A. Qurthobi, A. B. K. Pambudi, D. Darmawan, and R. F. Iskandar, "Correlation between Battery Voltage under Loaded Condition and Estimated State of Charge at Valve-Regulated Lead Acid Battery on Discharge Condition using Open Circuit Voltage Method," International Journal of Power Electronics and Drive Systems (IJPEDS), vol. 9 , p. $357,2018$.

[13] H.-G. Schweiger, O. Obeidi, O. Komesker, A. Raschke, M. Schiemann, C. Zehner, M. Gehnen, M. Keller, and P. Birke, "Comparison of several methods for determining the internal resistance of lithium ion cells," Sensors, vol. 10, pp. 5604-5625, 2010.

[14] H. Rahimi-Eichi and M.-Y. Chow, "Adaptive parameter identification and state-of-charge estimation of lithium-ion batteries," in IECON 2012-38th Annual Conference on IEEE Industrial Electronics Society, pp. 4012-4017, 2012.

[15] K. S. Ng, C.-S. Moo, Y.-P. Chen, and Y.-C. Hsieh, "Enhanced coulomb counting method for estimating state-ofcharge and state-of-health of lithium-ion batteries," Applied energy, vol. 86, pp. 1506-1511, 2009.

[16] S. Lee, J. Kim, J. Lee, and B. H. Cho, "State-of-charge and capacity estimation of lithium-ion battery using a new open-circuit voltage versus state-of-charge," Journal of Power Sources, vol. 185, pp. 1367-1373, 2008.

[17] S. Sepasi, L. Roose, and M. Matsuura, "Extended kalman filter with a fuzzy method for accurate battery pack state of charge estimation," Energies, vol. 8, pp. 5217-5233, 2015.

[18] J. Xie, W. Li, and Y. Hu, "Aviation lead-acid battery state-of-health assessment using PSO-SVM technique," in 2014 IEEE 5th International Conference on Software Engineering and Service Science, 2014, pp. 344-347.

[19] M. A. C. Valdez, J. A. O. Valera, M. Jojutla, and O. Arteaga, "Estimating SOC in lead-acid batteries using neural networks in a microcontroller-based charge-controller," in The 2006 IEEE International Joint Conference on Neural Network Proceedings, 2006, pp. 2713-2719.

[20] L. Zhi, Z. Peng, W. Zhifu, S. Qiang, and R. Yinan, "State of charge estimation for Li-ion battery based on extended Kalman filter," Energy Procedia, vol. 105, pp. 3515-3520, 2017.

[21] B. Xia, H. Wang, M. Wang, W. Sun, Z. Xu, and Y. Lai, "A new method for state of charge estimation of lithium-ion battery based on strong tracking cubature kalman filter," Energies, vol. 8, pp. 13458-13472, 2015.

[22] P. Manoharan, R. Mohankumar, K. Karthick, and R. Sowmya, "SoC Estimation and Monitoring of Li-ion Cell using Kalman-Filter Algorithm," Indonesian Journal of Electrical Engineering and Informatics (IJEEI), vol. 6, pp. 418-427, 2018.

[23] R. Xiao, J. Shen, X. Li, W. Yan, E. Pan, and Z. Chen, "Comparisons of modeling and state of charge estimation for lithium-ion battery based on fractional order and integral order methods," Energies, vol. 9, p. 184, 2016.

[24] X. Chen, W. Shen, Z. Cao, and A. Kapoor, "A novel approach for state of charge estimation based on adaptive switching gain sliding mode observer in electric vehicles," Journal of Power Sources, vol. 246, pp. 667-678, 2014.

[25] Z. Song, J. Hou, H. Hofmann, J. Li, and M. Ouyang, "Sliding-mode and Lyapunov function-based control for battery/supercapacitor hybrid energy storage system used in electric vehicles," Energy, vol. 122, pp. 601-612, 2017.

[26] R. Yamin and A. Rachid, "Modeling and simulation of a lead-acid battery packs in matlab/simulink: Parameters identification using extended kalman filter algorithm," in 2014 UKSim-AMSS 16th International Conference on Computer Modelling and Simulation, pp. 363-368, 2014.

[27] X. Li, J. Jiang, C. Zhang, L. Y. Wang, and L. Zheng, "Robustness of SOC estimation algorithms for EV lithium-ion batteries against modeling errors and measurement noise," Mathematical Problems in Engineering, vol. 2015, 2015.

[28] F. Zhang, G. Liu, and L. Fang, "A battery State of Charge estimation method with extended Kalman filter," in 2008 IEEE/ASME International Conference on Advanced Intelligent Mechatronics, 2008, pp. 1008-1013.

[29] C. Piao, Z. Sun, Z. Liang, and C. Cho, "SOC estimation of lead-acid batteries based on UKF," in 2010 International Conference on Electrical and Control Engineering, 2010, pp. 1968-1972.

[30] H. Dai, X. Wei, and Z. Sun, "Design and implementation of a UKF-based SOC estimator for LiMnO2 batteries used on electric vehicles," Przeglad Elektrotechniczny, vol. 88, pp. 57-63, 2012.

[31] Y. Tian, B. Xia, M. Wang, W. Sun, and Z. Xu, "Comparison study on two model-based adaptive algorithms for SOC estimation of lithium-ion batteries in electric vehicles," Energies, vol. 7, pp. 8446-8464, 2014.

[32] R. Xiong, H. He, F. Sun, and K. Zhao, "Evaluation on state of charge estimation of batteries with adaptive extended Kalman filter by experiment approach," IEEE Transactions on Vehicular Technology, vol. 62, pp. 108-117, 2013.

[33] Z. Chen, X. Li, J. Shen, W. Yan, and R. Xiao, "A novel state of charge estimation algorithm for lithium-ion battery packs of electric vehicles," Energies, vol. 9, p. 710, 2016.

[34] Z. Deng, L. Yang, Y. Cai, and H. Deng, "Online identification with reliability criterion and state of charge estimation based on a fuzzy adaptive extended Kalman filter for lithium-ion batteries," Energies, vol. 9, p. 472, 2016.

[35] H. Wang, W. Wang, L. Cui, H. Sun, J. Zhao, Y. Wang, and Y. Xue, "A hybrid multi-objective firefly algorithm for big data optimization," Applied Soft Computing, vol. 69, pp. 806-815, 2018. 


\section{BIOGRAPHIES OF AUTHORS}

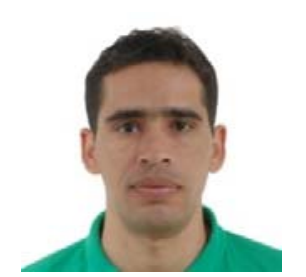

Maamar Souaihia received his engineering bachelor degree in electronics and instrumentation from the University of Hassiba Benbouali of Chlef in 2010. He obtained his master degree in the industrial computing from the University of Mostaganem in 2014. He is preparing his $\mathrm{PhD}$ thesis on the subject analysis and diagnosis batteries in Photvoltaic systems Since 2014 in the Department of Electrical Engineering at the University of Chlef.

E-mail:maa.souaihia@gmail.com

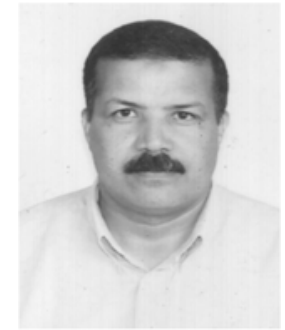

Bachir Belmadani received the B.S. degree in Electrical Engineering from the Mohamed Boudiaf University of Science and Technology, Oran, Algeria, in 1985, and the PhD degree from the Paul Sabatier University of Toulouse, France, in 1989. He is currently Professor of Power Electronics in Chlef University, Ouled Fares, Algeria. He is the Director of the Laboratory of Electrical Engineering and Renewable Energy and the Vice Rector for external relations with Chlef University. His research interests include power electronics, electrical drives and renewable energies. He has authored several international publications. His current research interests include power electronics, thermal and non-thermal plasmas and their applications, modeling and simulation of converters, field-programmable gate array, DSP, and intelligent control.

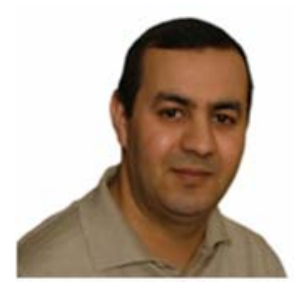

Rachid Taleb received the M.S. degree in electrical engineering from the Hassiba Benbouali University, Chlef, Algeria, in 2004 and the Ph.D. degree in electrical engineering from the Djillali Liabes University, Sidi Bel-Abbes, Algeria, in 2011. Currently he is a professor with the Department of Electrical Engineering, Hassiba Benbouali University. He is a team leader in the LGEER Laboratory (Laboratoire Génie Electrique et Energies Renouvelables). His research interest includes intelligent control, heuristic optimization, control theory of converters and converters for renewable energy sources. 